\title{
Strategies for glucose control in people with type 1 diabetes
}

Boiroux, Dimitri; Finan, Daniel Aaron; Jørgensen, John Bagterp; Poulsen, Niels Kjølstad; Madsen, Henrik

\section{Published in:}

Proceedings of the 18th World Congress of the International Federation of Automatic Control (IFAC)

Link to article, DOI:

10.3182/20110828-6-IT-1002.03714

Publication date:

2011

Link back to DTU Orbit

Citation (APA):

Boiroux, D., Finan, D. A., Jørgensen, J. B., Poulsen, N. K., \& Madsen, H. (2011). Strategies for glucose control in people with type 1 diabetes. In Proceedings of the 18th World Congress of the International Federation of Automatic Control (IFAC) https://doi.org/10.3182/20110828-6-IT-1002.03714

\section{General rights}

Copyright and moral rights for the publications made accessible in the public portal are retained by the authors and/or other copyright owners and it is a condition of accessing publications that users recognise and abide by the legal requirements associated with these rights.

- Users may download and print one copy of any publication from the public portal for the purpose of private study or research.

- You may not further distribute the material or use it for any profit-making activity or commercial gain

- You may freely distribute the URL identifying the publication in the public portal 


\title{
Strategies for Glucose Control in People with Type 1 Diabetes *
}

\author{
Dimitri Boiroux* Daniel A. Finan* John Bagterp Jørgensen* \\ Niels Kjølstad Poulsen* Henrik Madsen* \\ * Department of Informatics and Mathematical Modeling, \\ Technical University of Denmark, DK-2800 Kgs Lyngby, Demmark \\ (e-mail: $\{$ dibo,dafi,jbj,nkp,hm\}@imm.dtu.dk)
}

\begin{abstract}
In this paper we apply a robust feedforward-feedback control strategy to people with type 1 diabetes. The feedforward controller consists of a bolus calculator which compensates the disturbance coming from meals. The feedback controller is based on a linearized description of the model describing the patient. We minimize the risk of hypoglycemia by introducing a time-varying glucose setpoint based on the announced meal size and the physiological model of the patient. The simulation results are based on a virtual patient simulated by the Hovorka model. They include the cases where the insulin sensitivity changes, and mismatches in meal estimation. They demonstrate that the designed controller is able to achieve offset-free control when the insulin sensitivity change, and that having a time-varying reference signal enables more robust control of blood glucose in the cases where the meal size is known, but also when the ingested meal does not match the announced one.
\end{abstract}

\section{INTRODUCTION}

The World Health Organization [2009] estimates that more than 220 million people worldwide have diabetes. This number is likely to double by 2030 . In the USA, the budget for diabetes represents $10 \%$ of the health care budget, i.e. more than 130 billion dollars (132 billion dollars in 2002).

For healthy people, the blood glucose is tightly held at around $90 \mathrm{mg} / \mathrm{dL}$ (or $5 \mathrm{mmol} / \mathrm{L}$ ). Diabetes is a chronic disease characterized by an insufficient production of insulin and/or a decrease in its effectiveness. Therefore, people with diabetes tend to have a too high blood glucose level, also called hyperglycemia. Long periods of hyperglycemia can lead to complications like nerve diseases, kidney diseases, or blindness. However, the dosing of insulin must be done carefully, because a too high dosage of insulin may lead to hypoglycemia, which has immediate effects, such as insulin shock, coma or even death.

In particular, people with type 1 diabetes must rely on injection of exogenous insulin to survive. The current insulin therapy for people with type 1 diabetes consists of the injection of slow acting insulin once a day and fast acting insulin several times per day, usually before mealtimes. The slow acting insulin is used to counteract the continuous glucose production from the liver. The fast acting insulin compensates the intake of carbohydrates $(\mathrm{CHO})$ during the meals. The decision on the amount of short and fast acting insulin is based on 3-4 blood glucose measurements per day.

Continuous glucose monitors (CGMs) can improve the insulin therapy. In addition, insulin pumps can be used to adjust the insulin infusion rate, and insulin pens can be

\footnotetext{
* Funding for this research as part of the DIACON project from the Danish Council for Strategic Research is gratefully acknowledged.
}

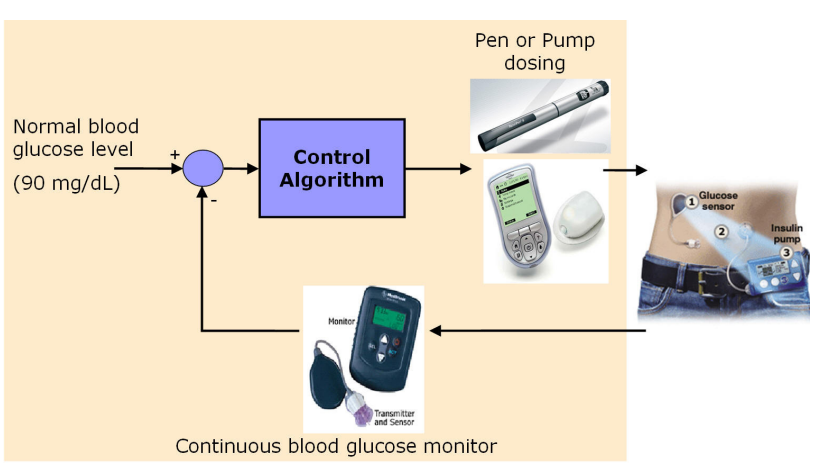

Fig. 1. Closed-loop glucose control. Glucose is measured subcutaneously using a continuous glucose monitor (CGM). Insulin is dosed either continuously by an insulin pump or discretely using an insulin pen.

used to administrate insulin boluses. These devices can be used in an artificial pancreas, which is described in Fig. 1. Various research groups work on aspects of control algorithms integrating the CGM and the insulin pump; see, e.g. Klonoff et al. [2009], Cobelli et al. [2009] and Magni et al. [2009].

In this paper we use a feedforward-feedback controller based on linear MPC. The feedforward controller computes the optimal bolus size to compensate the CHO ingested through meals. The feedback controller adjusts the basal insulin infusion rate.

The paper is structured as follows. Section 2 introduces the model used to simulate a virtual patient with type 1 diabetes. Section 3 describes the controller used to compute the optimal closed-loop insulin profiles. Section 4 presents the numerical results in the cases where the insulin sensitivity changes under fasting conditions, and 


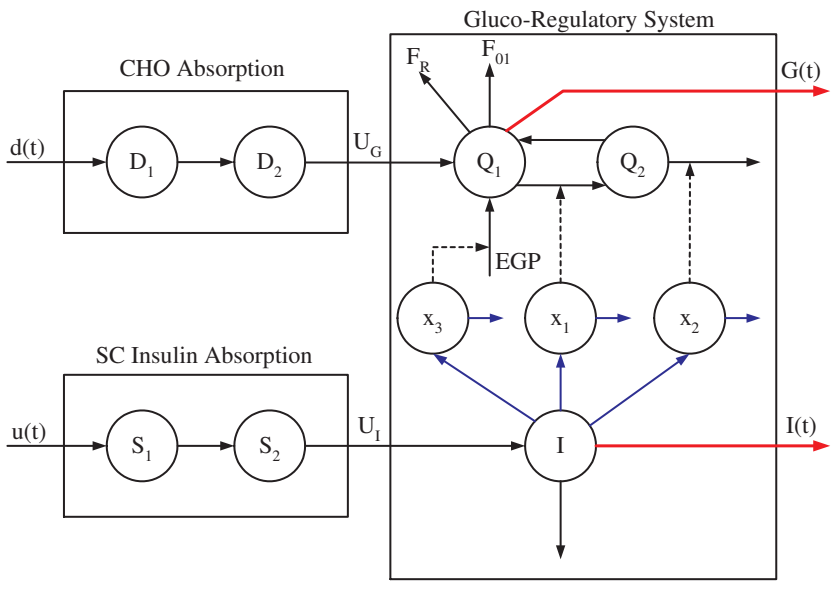

Fig. 2. Diagram of the Hovorka model.

in the case where the patient has one $75 \mathrm{~g}$ CHO meal. Conclusions are provided in Section 5.

\section{PHYSIOLOGICAL MODELS}

Several nonlinear physiological models have been developed to simulate virtual patients with type 1 diabetes, see e.g. Bergman et al. [1981], Hovorka et al. [2004], Dalla Man et al. [2007] and the review article written by Wilinska and Hovorka [2009]. In this paper, we use the Hovorka model to simulate people with type 1 diabetes. The models for CHO absorption, subcutaneous insulin absorption and the glucose-insulin dynamics for the Hovorka model are described in Fig. 2.

The delays associated to insulin and CHO absorption and the nonlinearities of the model limit the control quality, see e.g. Boiroux et al. [2010a]. Indeed, the time constant associated to $\mathrm{CHO}$ absorption is $\tau_{G}=40$ minutes, while the one associated to insulin absorption is $\tau_{I}=55$ minutes. The main source of nonlinearity in the Hovorka model arises from the bilinear term involving the action of insulin $x_{1}$ on glucose transport and the glucose in the main blood stream $Q_{1}$. This nonlinearity leads to an inaccurate description of linear models when the state of the system is not close to a steady state, e.g. during meals.

An other issue is that these models may be non-identifiable for some subjects (Pillonetto et al. [2003]).

\section{CONTROLLER DESIGN}

In this section we describe the feedforward-feedback controller used to compute the optimal insulin administration profiles. The controller is based on linear MPC, where the model is a linearized version of the Hovorka model. The reference signal is time-varying. The states of the system are estimated using a stationary Kalman filter with an integrated disturbance.

\subsection{Linear model}

We consider a system of ordinary differential equations (ODEs) in the form

$$
\begin{aligned}
\dot{x}(t) & =f(x(t), u(t), d(t)) \\
y(t) & =g(x(t))
\end{aligned}
$$

in which $x(t) \in \mathbf{R}^{n_{x}}$ describes the states of the system, $u(t) \in \mathbf{R}^{n_{u}}$ describes the manipulated variables (insulin infusion rate) $d(t) \in \mathbf{R}^{n_{d}}$ are disturbances (meals), and $y(t)$ depicts the measured output (blood glucose).

The system (1) can approximated by a linear state space system at a steady state $\left(x_{s s}, u_{s s}, d_{s s}\right)$. In this paper we choose the target value $\bar{G}=5 \mathrm{mmol} / \mathrm{L}$. The linear state space description in continuous time is

$$
\begin{aligned}
\dot{x}(t) & =A_{c} \delta x(t)+B_{c} \delta u(t)+E_{c} \delta d(t) \\
\delta y(t) & =C_{c} \delta x(t)
\end{aligned}
$$

In equation $(2), \delta x(t), \delta u(t), \delta d(t)$ and $\delta y(t)$ are deviation variables from the steady state $\left(x_{s s}, u_{s s}, d_{s s}\right)$, i.e.

$$
\begin{array}{ll}
\delta x(t)=x(t)-x_{s s} & \delta u(t)=u(t)-u_{s s} \\
\delta d(t)=d(t)-d_{s s} & \delta y(t)=y(t)-\bar{G}(t)
\end{array}
$$

and the time-invariant matrices $A_{c}, B_{c}, E_{c}$ and $C_{c}$ are

$$
\begin{aligned}
A_{c}=\frac{\partial f}{\partial x}\left(x_{s s}, u_{s s}, d_{s s}\right) & B_{c}=\frac{\partial f}{\partial u}\left(x_{s s}, u_{s s}, d_{s s}\right) \\
E_{c}=\frac{\partial f}{\partial d}\left(x_{s s}, u_{s s}, d_{s s}\right) & C_{c}=\frac{\mathrm{d} g}{\mathrm{~d} x}\left(x_{s s}\right)
\end{aligned}
$$

We now assume a zero-order hold parametrization of the controlled input $u$ and the disturbance $d$ with the sampling time $T_{s}=5 \mathrm{~min}$. Under this assumption, the continuoustime linear state space system (2) is equivalent to the deterministic linear discrete-time state space description

$$
\begin{aligned}
\delta x_{k+1} & =\bar{A} \delta x_{k}+\bar{B} \delta u_{k}+\bar{E} \delta d_{k} \\
y_{k} & =\bar{C} x_{k}
\end{aligned}
$$

\subsection{Time-varying reference signal}

Boiroux et al. [2010b] demonstrates that a constant glucose reference signal usually leads to an overdose of insulin when the meal size becomes too large. A time-varying glucose setpoint has been extensively used to reduce the risk of hypoglycemia, see e.g. Marchetti et al. [2006], GarciaGabin et al. [2008] and Eren-Oruklu et al. [2009]. It has also been noticed that the optimal insulin administration profile in the case where the meals are announced at mealtime only is close to a bolus-like profile (see Boiroux et al. [2010c] and Fig. 3). Consequently, the insulin administration can be separated between

- The basal insulin, which must compensate for endogenous glucose production. It must be adjusted to reject disturbances caused by changes in physiological parameters, e.g. changes in the insulin sensitivity (feedback control) 

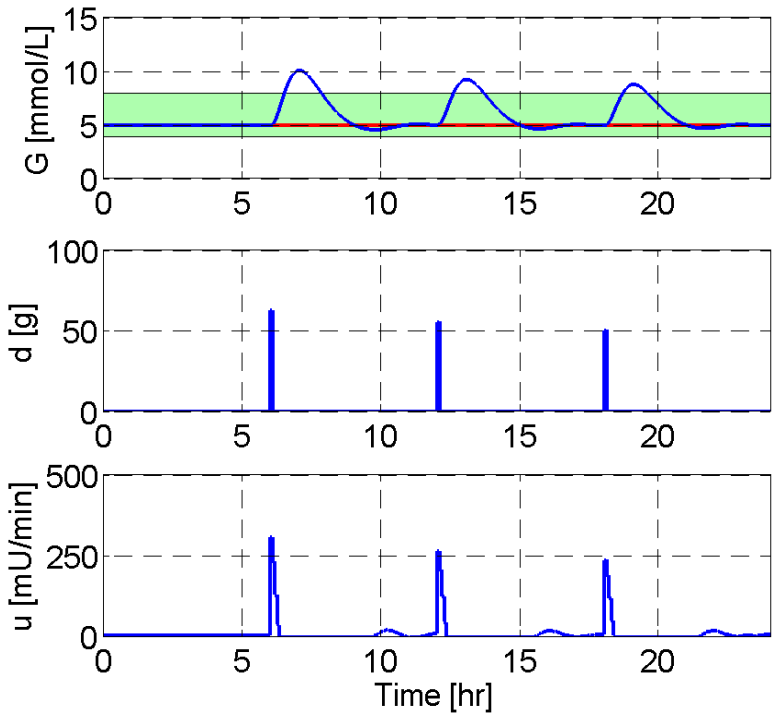

Fig. 3. Glucose profile (top), meal disturbances (middle) and optimal insulin administration profile (bottom) with meal announcement at meal time. Most insulin is taken in bolus like form at meal time.

- Insulin boluses, which are used to limit postprandial hyperglycemic event. The size of the bolus depends on the meal size announced by the patient (feedforward control)

The control strategy described above is a feedforwardfeedback control strategy similar to the one described in Marchetti et al. [2008]. When a meal is announced to the controller, the optimal bolus and the optimal postprandial blood glucose trajectory are computed by solving the univariate constrained optimization problem

$$
\begin{array}{ll}
\min _{u^{\text {bolus }}} & \psi=\frac{1}{2} \sum_{k=0}^{N-1}\left\|y_{k+1}^{0}-\bar{G}\right\|_{2}^{2} \\
\text { s.t. } & \dot{x}^{0}(t)=f\left(x^{0}(t), u_{k}, d_{k}\right) \quad t \in\left[t_{k}, t_{k+1}[\right. \\
& x_{0}^{0}=x_{s s} \\
& u_{0}^{0}=u_{s s}+u^{\text {bolus }} \\
& u_{k}^{0}=u_{s s}, \quad k=1,2, \ldots, N-1 \\
& y_{k}^{0}=C x_{k} \\
& y_{k}^{0} \geq \bar{G}
\end{array}
$$

In other words, we want to find the optimal bolus such that the reference signal is above the desired glucose target $\bar{G}=5 \mathrm{mmol} / \mathrm{L}$ for all times. In this case, the predictions on the future states of the system are made using the continuous-time nonlinear model.

The solution of (6) gives the reference insulin profile $u_{k}^{0}$, the reference states $x_{k}^{0}$ and the reference blood glucose setpoint $y_{k}^{0}$. Thus, we introduce the deviation variables from the reference state $\delta y_{k}, \delta x_{k}$ and $\delta u_{k}$ such that

$$
y_{k}=y_{k}^{0}+\delta y_{k} \quad x_{k}=x_{k}^{0}+\delta x_{k} \quad u_{k}=u_{k}^{0}+\delta u_{k}
$$
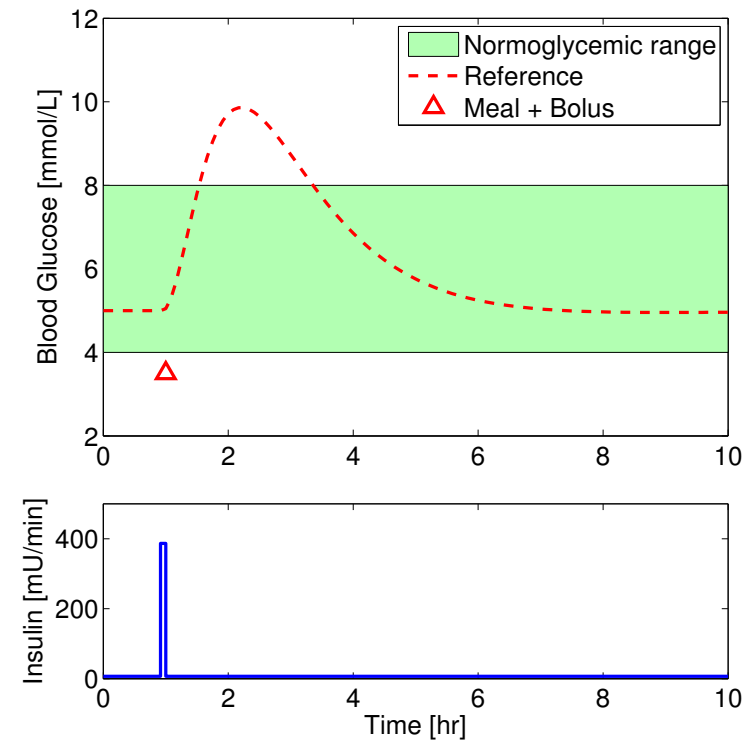

Fig. 4. Example of time-varying reference signal for the blood glucose. The meal size is $50 \mathrm{~g}$ CHO.

Fig. 4 gives an example of reference signal in the case where the patient has a $50 \mathrm{~g}$ CHO meal.

\subsection{Offset-free $M P C$}

Accordingly to Pannocchia and Rawlings [2003], offset-free control can be achieved by augmenting the state vector with an integrated disturbance $z_{k}$. In a fasting state, the stochastic linear discrete-time augmented state space description is

$$
\begin{aligned}
{\left[\begin{array}{c}
\delta x \\
z
\end{array}\right]_{k+1} } & =\left[\begin{array}{cc}
\bar{A} & B_{z} \\
0 & I
\end{array}\right]\left[\begin{array}{c}
\delta x \\
z
\end{array}\right]_{k}+\left[\begin{array}{c}
\bar{B} \\
0
\end{array}\right] \delta u_{k}+\left[\begin{array}{l}
\xi \\
\zeta
\end{array}\right]_{k} \\
\delta y_{k} & =\left[\begin{array}{ll}
\bar{C} & C_{z}
\end{array}\right]\left[\begin{array}{c}
\delta x \\
z
\end{array}\right]_{k}+w_{k}
\end{aligned}
$$

in which $B_{z} \in \mathbf{R}^{n_{x} \times n_{z}}$, and $C_{z} \in \mathbf{R}^{n_{y} \times n_{z}}$. The processes $\xi_{k}, \zeta_{k}$ and $w_{k}$ are zero-mean white noise processes, and we assume that $\xi_{k}$ and $\zeta_{k}$ are uncorrelated. For convenience, we will denote

$$
\begin{aligned}
A & =\left[\begin{array}{cc}
\bar{A} & B_{z} \\
0 & I
\end{array}\right] B=\left[\begin{array}{c}
\bar{B} \\
0
\end{array}\right] E=\left[\begin{array}{c}
\bar{E} \\
0
\end{array}\right] C=\left[\begin{array}{ll}
\bar{C} & C_{z}
\end{array}\right] \\
\underline{x}_{k} & =\left[\begin{array}{c}
\delta x \\
z
\end{array}\right]_{k}
\end{aligned}
$$

and we define the time-invariant covariance matrices

$$
R_{1}=\operatorname{Cov}\left(\left[\begin{array}{l}
\xi \\
\zeta
\end{array}\right]_{k}\right) \quad R_{2}=\operatorname{Cov}\left(v_{k}\right)
$$

We set $\operatorname{Var}\left[\xi_{k}\right]=0$. Hence, the variances of the white noise processes $\zeta_{k}$ and $v_{k}$ are tuning parameters. For the simulations we set 


$$
\operatorname{Var}\left[\zeta_{k}\right]=0.7^{2} \quad R_{2}=0.5^{2}
$$

The integrated disturbance process $z_{k}$ can be added either to the insulin infusion, or to the meal ingestion, or the the blood glucose measurements. In this paper, we choose to add the disturbance to the insulin infusion, i.e.

$$
B_{z}=\bar{B}, \quad C_{z}=0
$$

It must be pointed out that the reference glucose trajectory determined in section 3.2 already includes the effects of the meal intake and the associated bolus. Consequently, the one-step ahead prediction for the state vector $\delta \hat{x}_{k+1 \mid k}$ is

$$
\delta \underline{\hat{x}}_{k+1 \mid k}=A \delta \underline{\hat{x}}_{k \mid k}+B \delta u_{k}
$$

and the filtered state $\delta \underline{\hat{x}}_{k+1 \mid k+1}$ is computed as

$$
\begin{aligned}
e_{k+1} & =\delta y_{k+1}-C \delta \underline{\hat{x}}_{k+1 \mid k} \\
\delta \underline{\hat{x}}_{k+1 \mid k+1} & =\delta \underline{\hat{x}}_{k+1 \mid k}+K e_{k+1}
\end{aligned}
$$

in which $K$ is the stationary Kalman gain. The one-step ahead prediction and the filtering step are expressed in terms of deviation variables from the reference signal.

\subsection{Linear MPC with soft output constraints}

At each time sample, it is required to solve an openloop constrained optimization problem. Let $N$ be the prediction horizon length. The linear problem with hard input constraints and soft output constraints at time $t_{k}$ is formulated as

$$
\begin{array}{ll}
\min _{\left\{u_{i}, v_{i}\right\}_{i=0}^{N-1}} \phi=\frac{1}{2} \sum_{i=0}^{N-1}\left\|\delta \hat{y}_{k+i+1 \mid k}\right\|_{2}^{2}+ \\
& \lambda\left\|\Delta u_{k+i}\right\|_{2}^{2}+\kappa\left\|v_{i}\right\|_{2}^{2} \\
\text { s.t. } \quad & \delta \underline{\hat{x}}_{k+i+1 \mid k}=A \delta \underline{\hat{x}}_{k+i \mid k}+B u_{k+i} \\
& \delta \hat{y}_{k+i \mid k}=C \delta \underline{\hat{x}}_{k+i \mid k} \\
& u_{\min } \leq \delta u_{k+i} \leq u_{\max } \\
& \Delta u_{\min } \leq \Delta u_{k+i} \leq \Delta u_{\max } \\
& y_{\min }-r_{k+i}-\delta \hat{y}_{k+i \mid k} \leq v_{i} \\
& v_{i} \geq 0
\end{array}
$$

The slack variables $v_{i}$ are introduced to penalize hypoglycemia. The hard input constraints $(15 \mathrm{~d}-15 \mathrm{e})$ limit the insulin infusion rate and the increment of the insulin infusion rate respectively. The penalty term $\kappa\left\|v_{i}\right\|_{2}^{2}$ is used to avoid hypoglycemia and the penalty term $\lambda\left\|\Delta u_{i}\right\|_{2}^{2}$ prevents the insulin infusion rate from varying too aggressively.

For the simulations we choose $N=120$, i.e. a 10 hour prediction horizon, such that the computed optimal insulin profile is similar to the one in the case where the prediction horizon is infinite. Thus, the prediction horizon is not considered as a tuning parameter. The tuning parameters of (15) are the weights $\lambda$ and $\kappa$.

For the simulations we choose

$$
u_{\text {min }}=-\frac{u_{s s}}{2}, \quad \lambda=400, \quad \kappa=100
$$

The choice of $u_{\text {min }}=-\frac{u_{s s}}{2}$ instead of $u_{\text {min }}=-u_{s s}$ does not allow the controller to switch off the insulin pump. Instead, switching off the pump can be implemented as a safety layer in case of an (upcoming) hypoglycemic event.

\section{NUMERICAL RESULTS}

In this section, we use the Hovorka model and the described linear MPC algorithm to compute the optimal insulin administration profiles for people with type 1 diabetes. We consider two cases:

- A 36 hour simulation with a decrease in insulin sensitivity by $50 \%$ under fasting conditions. We both consider the case where the sensor is noise-free, and the case where the sensor is affected by white noise.

- A 24 hour simulation with one $75 \mathrm{~g}$ CHO meal. The meal is given 6 hours after the beginning of the simulation. We consider the cases where the correct meal size is announced, the meal size is underestimated by $50 \%$, the meal size is overestimated by $50 \%$ and the meal is not announced at all.

For the first case, the insulin sensitivity is changed by modifying the insulin sensitivities for the three insulin action compartments after 1 hour. The insulin sensitivities are described by the parameters $S_{I, 1}, S_{I, 2}$ and $S_{I, 3}$ in the Hovorka model (the model is described in Hovorka et al. [2004] and Boiroux et al. [2010b]). A decrease by $33 \%$ of these parameters will give a new insulin infusion basal rate

$$
\tilde{u}_{s s}=1.5 u_{s s}
$$

We assume that the noise process of the glucose sensor is a zero-mean white noise process which follows a Gaussian distribution with the standard deviation

$$
\sigma=0.5 \mathrm{mmol} / \mathrm{L}
$$

Fig. 5 illustrates the blood glucose and the insulin profiles in the case where a change in the insulin sensitivity occurs while the patient is fasting, with and without sensor noise (5(a) and 5(a)). The insulin infusion rate increases to reject the disturbance caused by the decrease in insulin sensitivity. In the uncontrolled case where the basal insulin infusion rate is not adjusted, the blood glucose tends to a new steady state in the hyperglycemic range.

Fig. 6 shows the insulin and blood glucose profiles in the case where the patient has a $75 \mathrm{~g}$ CHO meal, but the meal is not announced to the controller. In that case, a severe hypoglycemia cannot be avoided. A similar hypoglycemic event occurs if we allow to switch off the insulin pump and if we use noise-free blood glucose measurements instead (results not shown). 

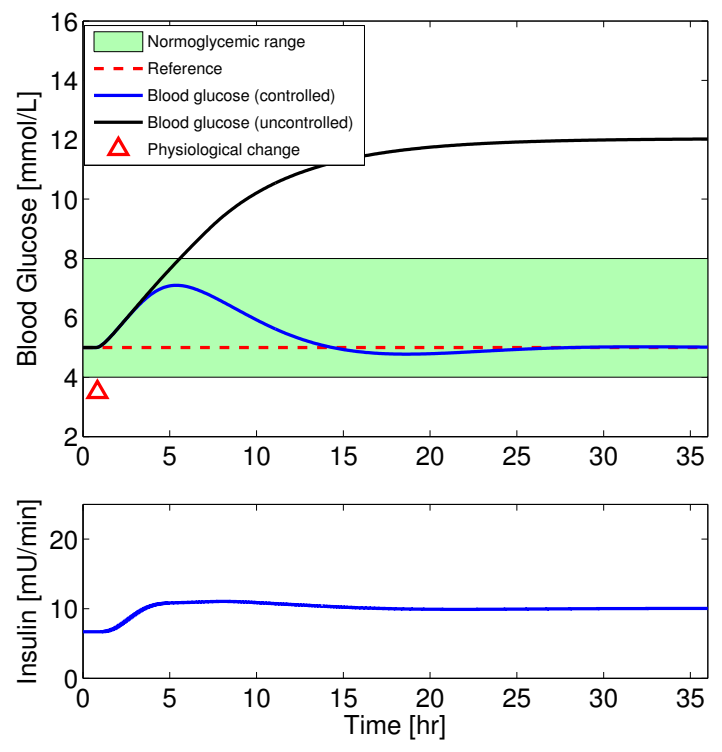

(a) Noise-free sensor
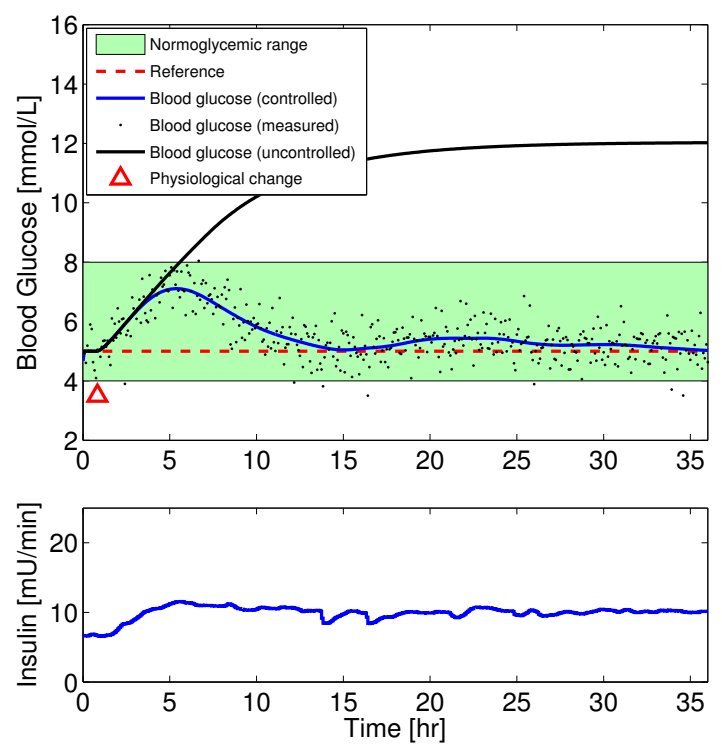

(b) Noisy sensor

Fig. 5. Insulin and blood glucose profile in the case where the insulin sensitivity decreases by $50 \%$ after 1 hour.

Fig. 7 illustrates the blood glucose and the insulin profiles in the case where the patient has a $75 \mathrm{~g}$ CHO meal. For the case where the exact meal size is announced (Fig. $7(\mathrm{a}))$, the insulin infusion rate remains close to the basal rate. Consequently, the blood glucose follows tightly the glucose setpoint. For the case where the meal size is underestimated (Fig. 7(b)), the basal rate increases after the mealtime to compensate for the too low bolus. For the case where the meal size is overestimated (Fig. 7(c)), the insulin infusion rate is at the minimum after the meal to compensate for the too high bolus. No hypoglycemic events occur when a meal is announced. However, the postprandial blood glucose excursion is bigger when the meal size is underestimated by the patient.
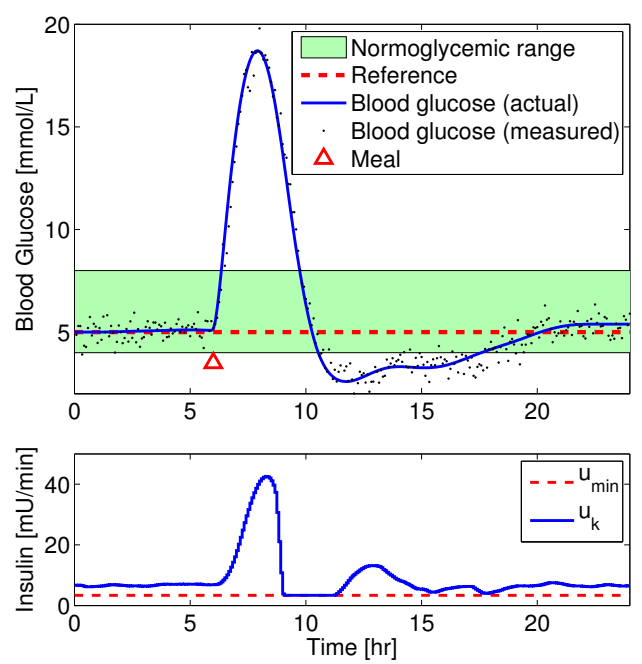

Fig. 6. Blood glucose and insulin profiles in the case where the meal is not announced.

These results show that reasonably good control can be obtained when a feedforward-feedback strategy is used. However, the main limitation of this strategy is that a fairly good nonlinear model description of the patient must be available.

\section{CONCLUSION}

In this paper, we described model predictive control based on a linearized version of the Hovorka model. The state is augmented with an integrated disturbance to ensure an offset-free control of the blood glucose. We use an optimal control algorithm to compute insulin administration profiles in the cases where the insulin sensitivity decreases by $50 \%$, and in the case where the patient has one $75 \mathrm{~g} \mathrm{CHO}$ meal. In the case where the patient has a meal, we consider the cases where the correct meal size is announced, the meal size is underestimated, the meal size is overestimated, and the meal is not announced at all. The results demonstrate that the control of blood glucose can be achieved without offset, and that a feedforwardfeedback control strategy is superior to a feedback control only, assuming that a good model description of the patient can be obtained.

\section{REFERENCES}

R. N. Bergman, L. S. Phillips, and C. Cobelli. Physiologic evaluation of factors controlling glucose tolerance in man: measurement of insulin sensitivity and beta-cell glucose sensitivity from the response to intravenous glucose. Journal of Clinical Investigation, 68(6):1456 - 1467, 1981.

D. Boiroux, D. A. Finan, N. K. Poulsen, H. Madsen, and J. B. Jørgensen. Nonlinear model predictive control for an artificial $\beta$-cell. In Recent Advances in Optimization and its Applications in Engineering, pages 299 - 308. Springer, 2010a.

D. Boiroux, D. A. Finan, N. K. Poulsen, H. Madsen, and J. B. Jørgensen. Optimal insulin administration for people with type 1 diabetes. In Proceedings of the 9th International Symposium on Dynamics and Control 
of Process Systems (DYCOPS 2010), pages $234-239$, 2010b.

D. Boiroux, D. A. Finan, N. K. Poulsen, H. Madsen, and J. B. Jørgensen. Implications and limitations of ideal insulin administration for people with type 1 diabetes. In UKACC International Conference on Control 2010 , pages 156 - 161, 2010c.

C. Cobelli, C. Dalla Man, G. Sparacino, L. Magni, G. De Nicolao, and B. P. Kovatchev. Diabetes: Models, signals, and control. IEEE Reviews in Biomedical Engineering, 2:54-96, 2009.

C. Dalla Man, R. Rizza, and C. Cobelli. Meal simulation model of the glucose-insulin system. IEEE Transactions on Biomedical Engineering, 54(10):1740-1749, 2007.

M. Eren-Oruklu, A. Cinar, L. Quinn, and D. Smith. Adaptive control strategy for regulation of blood glucose levels in patients with type 1 diabetes. Journal of Process Control, 19:1333 - 1346, 2009.

W. Garcia-Gabin, J. Vehí, J. Bondia, C. Tarín, and R. Calm. Robust sliding mode closed-loop glucose control with meal compensation in type 1 diabetes mellitus. In Proceedings of the 17th World Congress, The International Federation of Automatic Control, pages 4240 4245, 2008.

R. Hovorka, V. Canonico, L. J. Chassin, U. Haueter, M. Massi-Benedetti, M. O. Federici, T. R. Pieber, H. C. Schaller, L. Schaupp, T. Vering, and M. E. Wilinska. Nonlinear model predictive control of glucose concentration in subjects with type 1 diabetes. Physiological Measurement, 25:905-920, 2004.

D. C. Klonoff, C. Cobelli, B. Kovatchev, and H. C. Zisser. Progress in development of an artificial pancreas. Journal of Diabetes Science and Technology, 3:10021004, 2009.

L. Magni, D. M. Raimondo, C. Dalla Man, G. De Nicolao, B. P. Kovatchev, and C. Cobelli. Model predictive control of glucose concentration in type I diabetic patients: An in silico trial. Biomedical Signal Processing and Control, 4(4):338-346, 2009.

G. Marchetti, M. Barolo, L. Jovanovič, H. Zisser, and D. E. Seborg. An improved PID switching control strategy for type 1 diabetes. In 2006 International Conference of the IEEE Engineering in Medicine and Biology Society, pages 5041-5044, New York City, USA, 2006.

G. Marchetti, M. Barolo, L. Jovanovič, H. Zisser, and D. E. Seborg. A feedforward-feedback glucose control strategy for type 1 diabetes mellitus. Journal of Process Control, 18(2):149-162, 2008.

G. Pannocchia and J. B. Rawlings. Disturbance models for offset-free model-predictive control. AIChE Journal, 49(2):426-437, 2003.

G. Pillonetto, G. Sparacino, and C. Cobelli. Numerical non-identifiability regions of the minimal model of glucose kinetics: superiority of bayesian estimation. Mathematical Biosciences, 184:53 - 67, 2003.

M. E. Wilinska and R. Hovorka. Simulations models for in-silico testing of closed-loop glucose controllers in type i diabetes. Drug Discovery Today: Disease Models, 5(4): $289-298,2009$

World Health Organization. Diabetes (fact sheet no. 312), November 2009. WHO Web site: http://www.who.int/mediacentre/factsheets/fs312/en/.
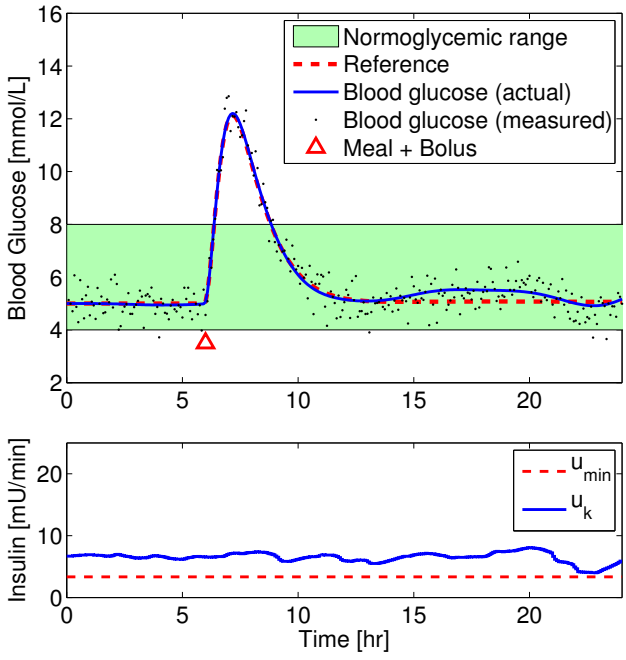

(a) Correct meal size
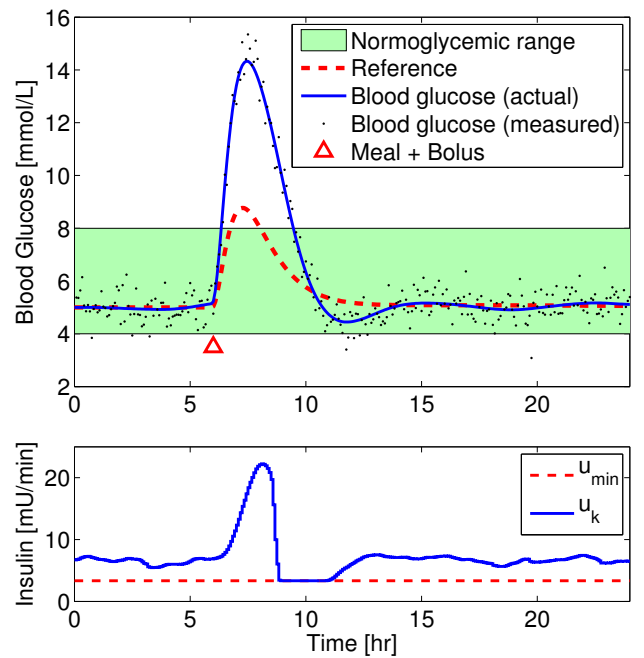

(b) Meal size underestimated by $50 \%$
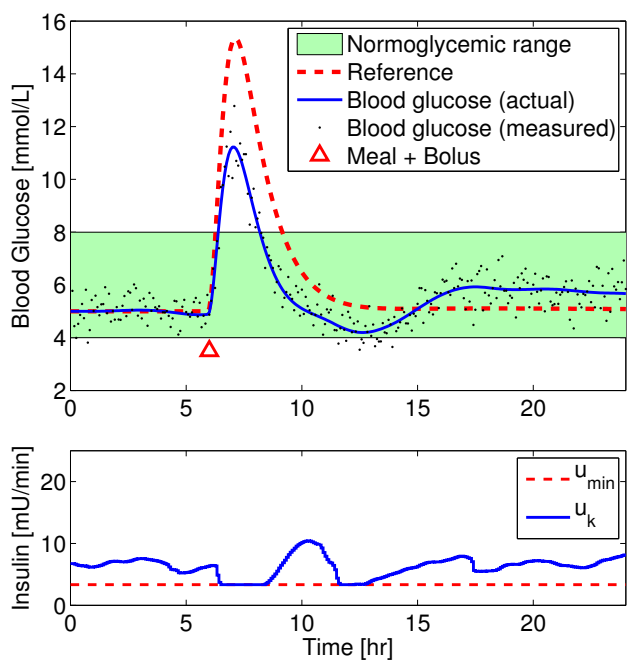

(c) Meal size overestimated by $50 \%$

Fig. 7. Blood glucose and insulin profiles for the 24 hour simulations with one $75 \mathrm{~g}$ CHO meal. 